\title{
Repeated cerebellar infarction in the affected nondominant vertebral artery distribution with reversible vertebral artery occlusion elicited by head tilt: illustrative case
}

\author{
Takanori Nozawa, MD, PhD, ${ }^{1,3}$ Kouichirou Okamoto, MD, PhD, ${ }^{2}$ Shinji Nakazato, MD, PhD, ${ }^{1}$ Kunio Motohashi, MD, ${ }^{1,3}$ \\ Tomoaki Suzuki, MD, PhD, ${ }^{3}$ Kotaro Morita, MD, PhD, ${ }^{1}$ Hideki Tashi, MD, PhD, ${ }^{4}$ Kei Watanabe, MD, PhD, ${ }^{4}$ Hitoshi Hasegawa, MD, PhD, ${ }^{3}$ \\ Masato Watanabe, MD, PhD, ${ }^{1}$ Hiroyuki Kawashima, MD, PhD, ${ }^{4}$ and Yukihiko Fujii, MD, PhD ${ }^{3}$
}

'Department of Neurosurgery, Kuwana Hospital, Niigata City, Japan; Departments of ${ }^{2}$ Translational Research and ${ }^{3}$ Neurosurgery, Brain Research Institute, Niigata University, Niigata City, Japan; and ${ }^{4}$ Division of Orthopedic Surgery, Department of Regenerative and Transplant Medicine, Graduate School of Medical and Dental Sciences, Niigata University, Niigata City, Japan

BACKGROUND Bow hunter's syndrome or stroke (BHS) is characterized by rotational vertebrobasilar insufficiency elicited by rotation of the neck. It is caused by dynamic and reversible occlusion of the vertebral artery (VA). Reversible symptoms of rotational vertebrobasilar insufficiency are described as bow hunter's syndrome, although brain infarction is rarely reported as bow hunter's stroke.

OBSERVATIONS A 70-year-old man experienced repeated cerebellar infarctions three times in the posterior inferior cerebellar artery (PICA) distribution of the nondominant right VA connecting the basilar artery. The onset of symptoms indicating cerebellar infarcts and the patient's head position changes were unrelated. Dynamic digital angiography (DA) revealed that the nondominant right VA was occluded by an osteophyte from the C4 vertebral body, and the right PICA branches were shown to be passing through the distal right VA from the left VA. These findings were observed when the patient's head was tilted to the right. An arterio-arterial embolic mechanism was suggested as the cause of repeated cerebellar infarctions.

LESSONS Transient nondominant VA occlusion has been rarely reported as a cause of BHS when the head is tilted. To confirm the diagnosis of BHS, additional head tilt is recommended when performing dynamic DA in patients with a cervical osteophyte.

https://thejns.org/doi/abs/10.3171/CASE2061

KEYWORDS repeated cerebellar infarction; bow hunter's syndrome; stroke; cervical osteophyte; transient vertebral artery occlusion; head tilt

Bow hunter's syndrome or stroke (BHS) is characterized by rotational vertebrobasilar insufficiency. This condition is elicited by neck rotation in most cases and by neck extension or flexion in some cases. $\mathrm{BHS}$ is caused by dynamic and reversible occlusion of the vertebral artery (VA) and a lack of collateral blood supply to the brainstem. ${ }^{1-4}$ Although the term "bow hunter's stroke" was coined by Sorensen for a patient with suspected brainstem infarction, ${ }^{5}$ reversible symptoms of rotational vertebrobasilar insufficiency are usually described as bow hunter's syndrome. ${ }^{4}$

BHS associated with recurrent cerebellar infarction and transient occlusion of the nondominant VA induced by head tilt has not been reported in the literature. In this study, we report a case involving an older man who experienced recurrent cerebellar infarctions three times in the last 2 years after undergoing a cervical left laminoplasty procedure that had been performed 5 years earlier. The infarctions occurred in the posterior inferior cerebellar artery (PICA) distribution of the affected nondominant VA, with no infarction occurring in any other region. Transient occlusion of the right $\mathrm{VA}$ by an osteophyte at the level of $\mathrm{C} 4$ was demonstrated during dynamic digital angiography (DA) when the patient's head was tilted to the right. No symptoms or additional infarction occurred after surgery with posterior fixation of the cervical spine (C3-C6).

\section{Illustrative Case}

A 70-year-old man underwent left laminoplasty at C3-C6 in a different hospital 5 years ago for ossification of the posterior

ABBREVIATIONS 3D-CTA = three-dimensional computed tomography angiography; $\mathrm{BHS}=$ bow hunter's syndrome or stroke; $\mathrm{DA}=$ digital angiography; DSA = digital subtraction angiography; $\mathrm{DWI}=$ diffusion-weighted imaging; $\mathrm{MRA}=$ magnetic resonance angiography; $\mathrm{MRI}=$ magnetic resonance imaging; $\mathrm{PICA}=$ posterior inferior cerebellar artery; $V A=$ vertebral artery.

INCLUDE WHEN CITING Published February 22, 2021; DOI: 10.3171/CASE2061.

SUBMITTED December 10, 2020. ACCEPTED December 17, 2020.

(c) 2021 The authors, CC BY-NC-ND 4.0 (http://creativecommons.org/licenses/by-nc-nd/4.0/). 
longitudinal ligament. He had a prominent medical history of cerebellar infarction in the lateral region of the right PICA distribution that was detected 2 years ago. For this he had started taking oral clopidogrel (75 $\mathrm{mg} /$ day). He was admitted to our hospital with complaints of the sudden onset of dizziness, nausea, and vomiting. Diffusion-weighted imaging (DWI) revealed an additional cerebellar infarction in the medial region of the right PICA distribution, and magnetic resonance angiography (MRA) revealed that the right $V A$ was occluded (Fig. $1 A, B, D$, and $E$ ). Three days later, repeated MRA showed recanalization of the nondominant right VA connecting the basilar artery (Fig. $1 \mathrm{C}$ and F). Echocardiography and 24-hour Holter monitor electrocardiography results were negative, and the etiology of his recurrent cerebellar infarction could not be elucidated. He was discharged from the hospital, and the following medications were prescribed without anticoagulants: aspirin (100 mg/day) and clopidogrel (75 mg/day).

Two months later, the man returned to our hospital with symptoms identical to those reported during the previous admission. DWI and MRA showed a new small cerebellar infarction in the right side of the inferior cerebellar vermis, with no infarction in any other region, as well as another right VA occlusion (Fig. 2A, B, D, and E). The right VA appeared patent on MRA on the 12th day after the second admission to our hospital (Fig. 2C and F). Three-dimensional computed tomography angiography (3D-CTA) showed that the right VA was laterally compressed by an osteophyte from the $\mathrm{C} 4$ vertebral body with no stenosis (Fig. 3B, arrow). Nineteen days later, during the man's second admission, he complained of symptoms similar to those in his first admission, including dizziness, nausea, and vomiting. MRA revealed an identical right VA occlusion with no new infarction on DWI. During subsequent dynamic digital subtraction angiography (DSA) and DA procedures with the patient's head in the neutral position, the nondominant right VA and the vermian and hemispheric branches of the right PICA were injected without stenosis (Fig. 4A-D). No severe stenosis or occlusion was revealed in the right VA when the neck was rotated to the right (Fig. 4E) or left (Fig. 4F) or when the neck was flexed or extended. However, the right VA was occluded by an osteophyte at the C4 level when his head was tilted to the right, which elicited no complaints of dizziness, nausea, or vomiting (Fig. 4G). A similar occlusion was not induced by head tilt to the left (Fig. 4H). No stenosis or occlusion was demonstrated in the dominant left VA on dynamic DA. The distal right VA was demonstrated in a retrograde fashion by injection of the left VA when the patient's head was tilted to the right (Fig. 4I), and both the vermian and hemispheric branches of the right PICA were subsequently opacified (Fig. $4 \mathrm{~J}$ and $\mathrm{K}$ ). The man was asymptomatic while his head was tilted to the right for more than 1 minute.

The patient denied that changes in the head or neck position caused the symptoms or the stroke. An arterio-arterial embolism from the transiently occluded right VA to the right PICA distribution was suggested as a cause of the repeated cerebellar infarction in this patient. He underwent posterior cervical fixation at C3-C6 at Niigata University Medical and Dental Hospital. After the posterior cervical fixation, both VAs were shown to operate continuously without stenosis from the
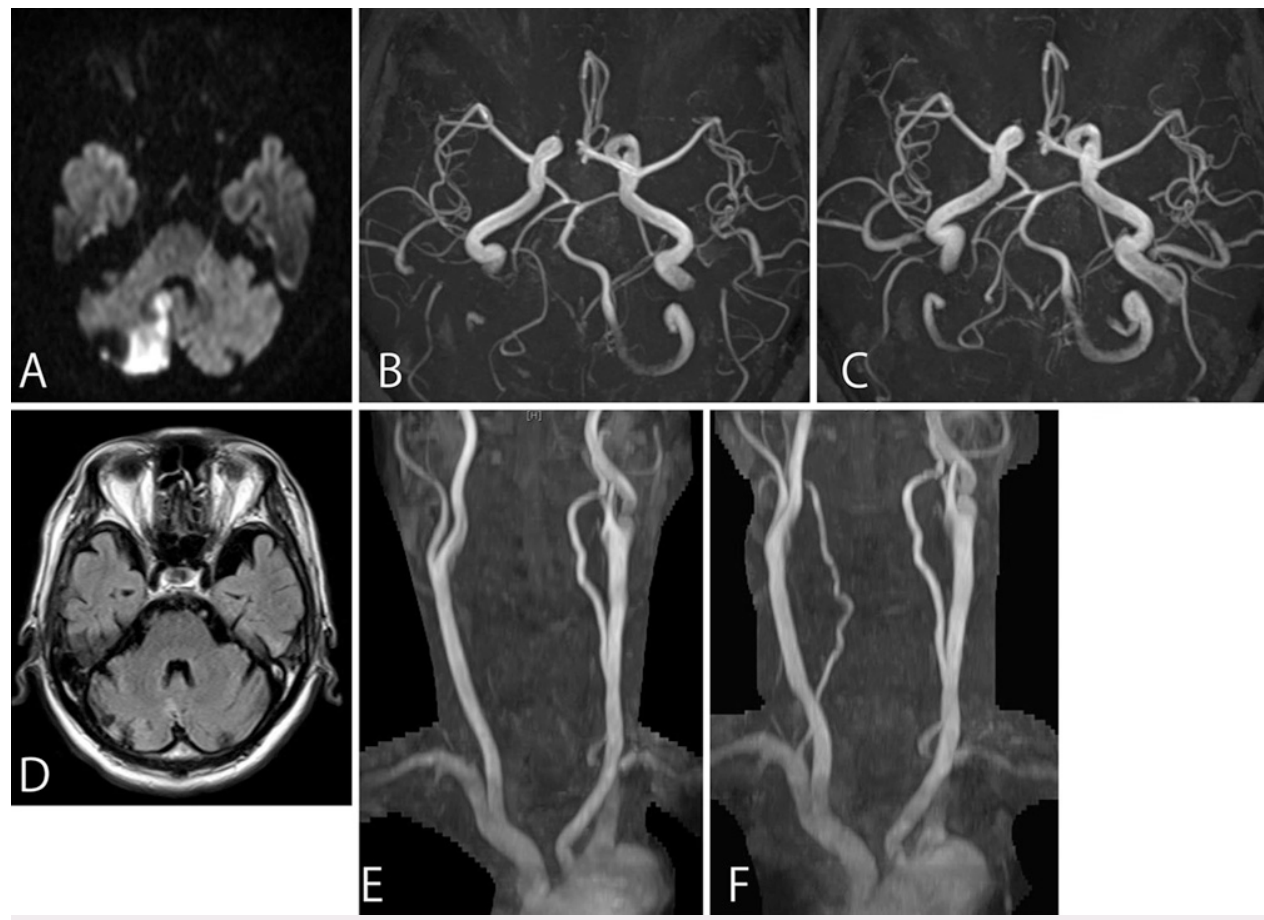

FIG. 1. MRI and MRA on the patient's first admission to our hospital (A, B, D, and E) and follow-up MRA at his first admission ( $\mathbf{C}$ and $\mathbf{F}$ ). $\mathbf{A}$ : DWI showing a high signal intensity lesion in the medial right PICA distribution. Brain (B) and cervical (E) MRA on admission. The right VA is not demonstrated. D: FLAIR image showing a previous right cerebellar infarction as a small hypointensity lesion in the lateral right PICA distribution. Brain (C) and cervical (F) MRA 3 days later. The nondominant right VA is observed from the origin to the junction of the left VA and basilar artery. FLAIR = fluid-attenuated inversion recovery. 

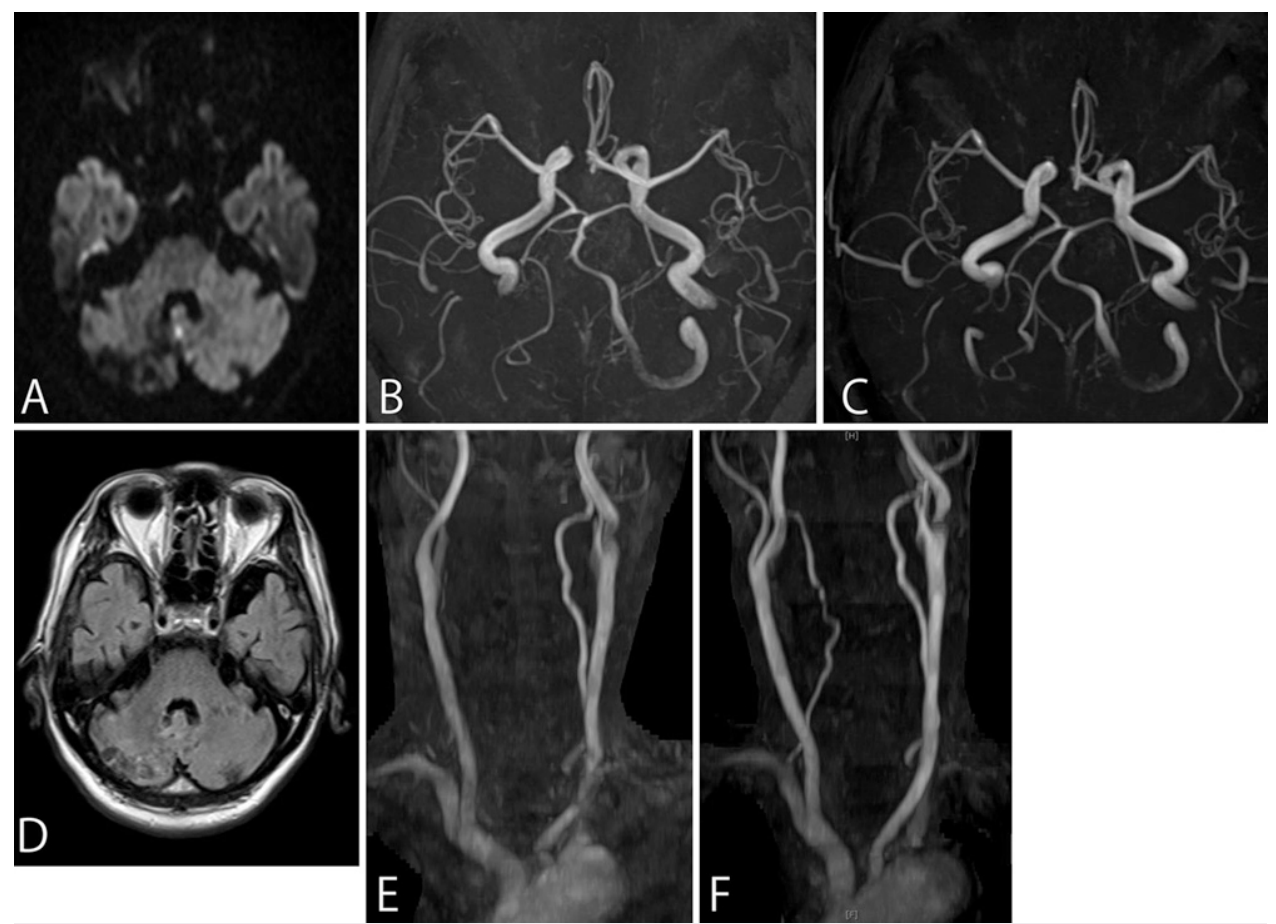

FIG. 2. MRI and MRA on the patient's second admission to our hospital (A, B, D, and E) and follow-up MRA at his second admission ( $\mathbf{C}$ and $\mathbf{F})$. A: DWI showing a small, high signal intensity lesion in the right side of the inferior cerebellar vermis. Brain (B) and cervical (E) MRA on admission. The right VA is not observed as it was on his previous admission (see Fig. 1B and E). D: FLAIR image showing past cerebellar infarcts in the right PICA distribution as hypointense lesions. Brain (C) and cervical (F) MRA 12 days later. The right VA appears again as previously demonstrated (see Fig. 1C and F).

origin to the basilar artery on 3D-CTA (Fig. 5). He had no symptoms, and no additional cerebellar infarction developed for 1 year 6 months after the surgery.

\section{Discussion}

In this patient, repeated cerebellar infarction was restricted to the PICA distribution with reversible occlusion of the ipsilateral

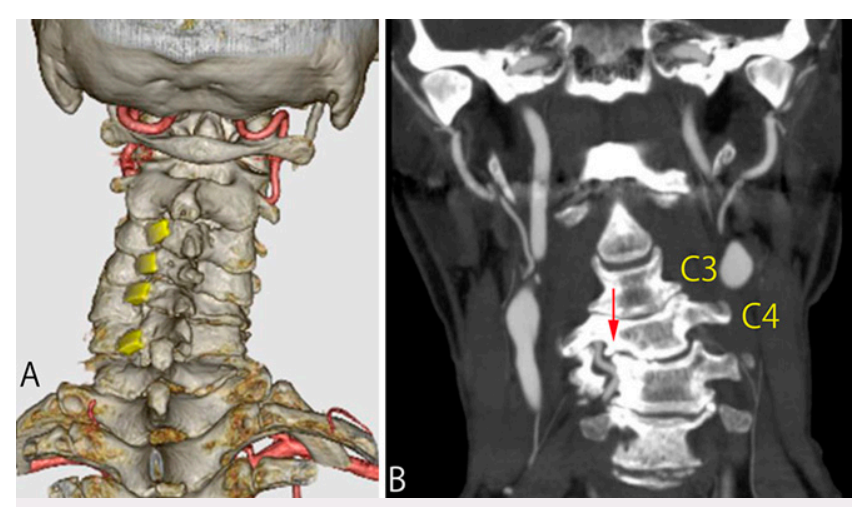

FIG. 3. 3D-CTA. A: Posterior view with volume-rendering method showing C3-C6 left laminoplasty performed at a previous clinic. B: Coronal reformatted image. An osteophyte of C4 (red arrow) compresses the right VA laterally without stenosis. nondominant right VA and was demonstrated three times on MRI and MRA in the past 2 years after a C3-C6 left laminoplasty that had been performed 5 years earlier. 3D-CTA showed an osteophyte of C4 compressing the right VA laterally without stenosis. On dynamic DA, occlusion of the right VA was demonstrated when the patient tilted his head to the right, although no severe stenosis or occlusion was observed in other head and neck positions. No stenosis or occlusion was shown in the dominant left VA. In this patient, without demonstration of the right $V A$ occlusion elicited by head tilt to the right, diagnosis of BHS could not be made, even after dynamic DA was performed. In elderly male patients (most $\geq 40$ years), reversible symptomatic VA stenosis/ occlusion caused by an osteophyte of the degenerative cervical vertebral body is one of the common characteristics in BHS when changing head and neck positions. ${ }^{1-6}$ In our patient, left laminoplasty of C3-C6 might have predisposed him to contralateral cervical osteophyte formation or growth at the C4 vertebral body.

BHS is the descriptive term for rotational vertebrobasilar insufficiency. This condition is elicited by neck rotation in most cases and by neck extension or, rarely, by neck flexion in some patients. BHS is caused by dynamic and reversible occlusion of the VA and a lack of collateral blood supply to the brainstem, either because of a hypoplastic or an absent contralateral VA or because of a deficient circle of Willis. ${ }^{4}$ Both VAs have adequate flow, and the patient remains asymptomatic when one VA is positionally occluded. ${ }^{7}$ In 1978 , Sorensen was the first to use the term "bow hunter's stroke" in a case of suspected brainstem infarction due to a VA injury, ${ }^{5}$ although brain infarction is rare in BHS. ${ }^{7-10}$ The suspected cause of cerebral or cerebellar infarction in BHS is 


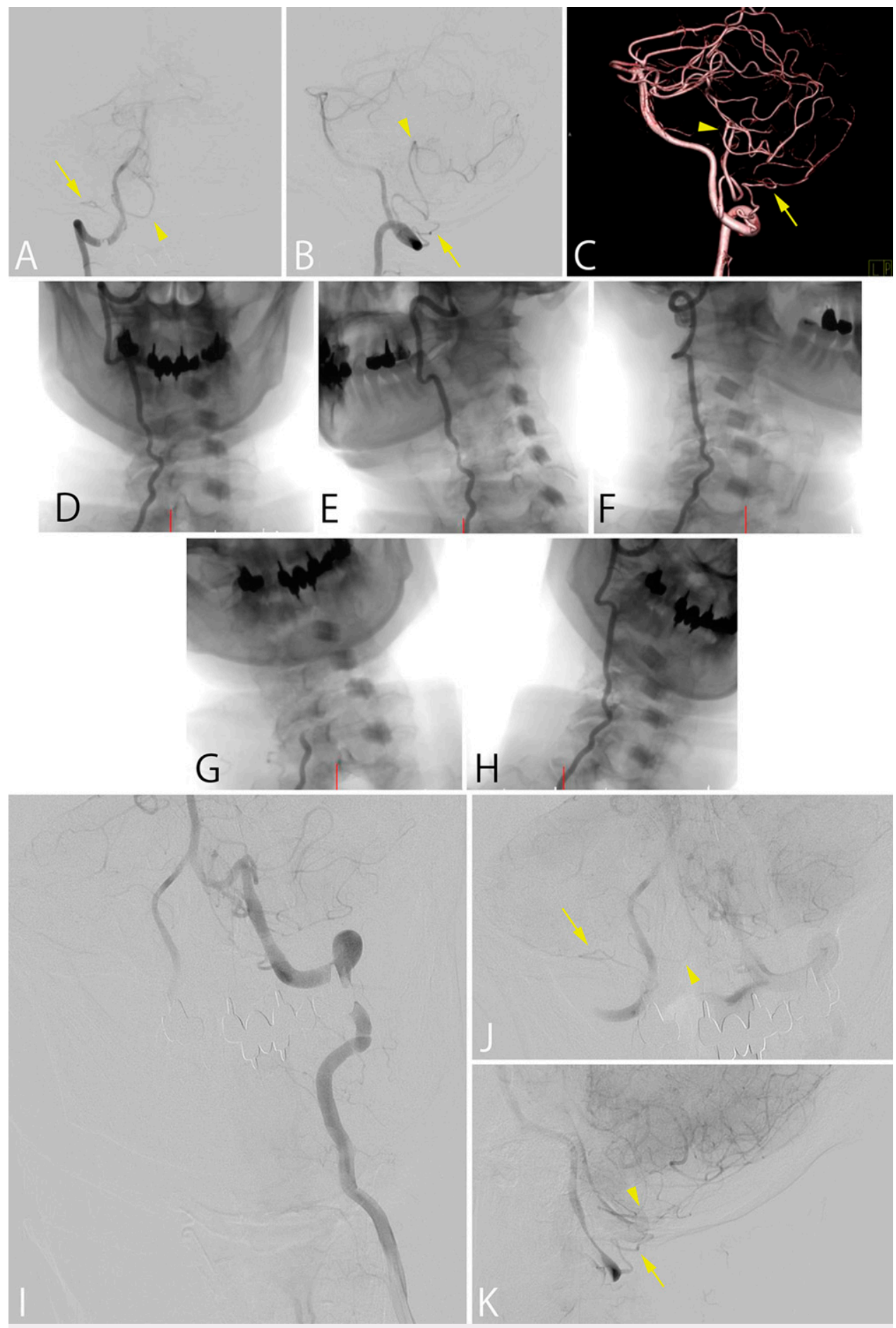

FIG. 4. Dynamic bilateral vertebral arteriography. A-C: DSA of the right VA at the neutral position of the head and neck. The hemispheric (arrows) and vermian (arrowheads) branches of the right PICA are demonstrated by injection of the right VA. A: Anterior-posterior view. B: Lateral view. C: Lateral view of 3D-rotation angiography with the volume-rendering method. D-H: Dynamic DA of the right VA. No stenosis is seen in the right VA at the neutral position (D), rotation of the neck to the right $(\mathbf{E})$ and to the left $(\mathbf{F})$, and head tilt to the left (H). Occlusion of the right VA is demonstrated at C4 level during head tilt to the right (G). I-K: Dynamic DSA of the left VA during head tilt to the right. I and J: Anterior-posterior view. K: Lateral view. The distal portion of the right VA is opacified in a retrograde fashion from the left VA (I), and both PICA hemispheric (arrows) and vermian (arrowheads) branches are subsequently shown ( $\mathbf{~}$ and $\mathbf{K})$. 


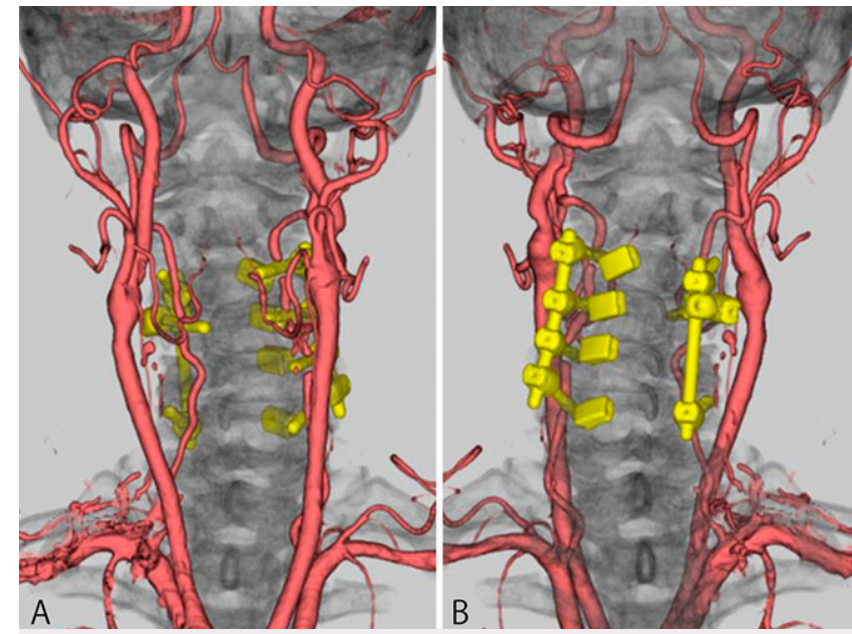

FIG. 5. 3D-CTA with volume-rendering method after C3-C6 posterior cervical fixation. A: Anterior view. B: Posterior view. Both VAs are demonstrated without stenosis.

hemodynamic impairment ${ }^{1-4,8}$ or arterio-arterial embolism from the affected VA..$^{7-10}$ In our patient, the hemodynamic mechanism for repeated cerebellar infarction in the right PICA distribution was less likely given that he was asymptomatic when the right VA was occluded at the C4 level. Moreover, the right intracranial VA and branches of the right PICA were demonstrated during dynamic DA of the left VA when the patient's head was tilted to the right. However, repeated cerebellar infarcts developed in the right PICA distribution in the 2 years after the C3-C6 left laminoplasty procedure that had been performed 5 years earlier; nonetheless, he denied that these events occurred during neck positional changes. Arterio-arterial embolism from the transiently occluded right VA was a likely mechanism for cerebellar infarction in this patient, although the precise mechanism remains unexplained for repeated infarction restricted to the ipsilateral PICA distribution.

BHS is caused by an atypical pattern of transient occlusion of a nondominant VA terminating in the PICA..$^{6,11-13}$ In addition, BHS that results from nondominant VA compression, similar to that in our patient, does not terminate in the PICA and has recently been reported in 20 cases. ${ }^{14}$ Among these cases, BHS caused by thromboembolism without $V A$ dissection was extremely rare in patients with degenerative cervical spondylosis., ${ }^{9,10,14}$ Such a condition was reported in two patients with wake-up stroke in the PICA distribution: ipsilateral in one patient and contralateral to the affected nondominant right $\mathrm{VA}$ in the other. ${ }^{9,10}$ The affected nondominant right VA was stenotic at $\mathrm{C} 4-\mathrm{C} 5$ or $\mathrm{C} 5-\mathrm{C} 6$ in the neutral position but completely occluded with head rotation to the right. 9,10 However, in our patient, repeated cerebellar infarction was not a wake-up stroke, and the nondominant right VA was not stenotic in the neutral head position (Fig. 4D), although complete occlusion of the right VA was induced by head tilt to the right (Fig. 4G). BHS induced by head tilt is an extremely rare condition. 6,15

Treatment for BHS includes conservative treatment and surgery. Conservative treatment includes the avoidance of head rotation, cervical collars, and/or antiplatelet or anticoagulation therapy.4,6,16 Conservative management with antiplatelet agents may be considered the first line of treatment for $\mathrm{BHS} .^{6}$ Dual-antiplatelet therapy with aspirin and clopidogrel was used in our patient. The combination of these antiplatelet agents synergistically inhibits platelet aggregation. ${ }^{17}$ In comparison with aspirin monotherapy, such dual-antiplatelet therapy reduces the rate of stroke occurrence from $11.7 \%$ to $8.2 \%$ in patients who have transient ischemic attack or minor stroke. ${ }^{17}$ However, repeated cerebellar infarction occurred in our patient while on the therapy. Some possible reasons for these repeated occurrences include the limited contribution of these antiplatelet agents because the affected nondominant VA showed no stenosis in the neutral head position and/or antiplatelet resistance to one or both antiplatelet agents. ${ }^{18}$ Nearly $50 \%$ of patients who receive conservative treatment are vulnerable to infarcts or have residual neurological deficits. ${ }^{16}$

Surgical intervention is proposed if medical treatment fails. ${ }^{14}$ All previously reported patients with BHS caused by nondominant VA compression required surgical management. ${ }^{14}$ Therefore, surgical treatment was considered in the current patient. An anterior approach is often used to remove osteophytes at C4-C6.1,2,4,9,10,14,19-21 PICA-toPICA bypass surgery has also been performed in a patient. ${ }^{22}$ Recently, successful endovascular treatment with angioplasty and stenting for contralateral VA stenosis was reported as an attempt to augment collateral flow. ${ }^{23}$ In a patient with transient occlusions of the VA by an osteophyte from a cervical vertebral body while changing neck positions and associated with repeated embolic infarction in the posterior circulation, a coil embolization procedure was performed at the distal portion of the affected VA to prevent further embolic infarction in the posterior circulation. ${ }^{24}$ The current patient underwent C3-C6 left laminoplasty for ossification of the posterior longitudinal ligament 5 years ago, and posterior fusion of C3-C6 was subsequently selected. After surgery, he experienced no additional symptoms or further cerebellar infarctions.

\section{Observations}

Repeated cerebellar infarction developed in the right PICA distribution in our patient. No VA stenosis was observed on 3D-CTA and DSA in the neutral head position, although DA revealed that the nondominant right VA was occluded by an osteophyte from the C4 vertebral body and that the right PICA branches passed through the distal right VA from the left VA without producing any symptoms when the patient's head was tilted to the right. Transient nondominant VA occlusion has been rarely reported as a cause of $\mathrm{BHS}$ when the head is tilted.

\section{Lessons}

In patients who show an association between repeated cerebellar infarction of unknown cause and degenerative cervical spondylosis, the occurrence of BHS should be considered even in the following instances: VAs are not stenotic in the neutral head position, a nondominant VA connects to the opposite VA and the basilar artery, and no characteristic symptoms are induced by head and neck positional changes. To gain further information, additional head tilt is recommended during a dynamic DA study for patients with suspected BHS.

\section{Acknowledgments}

We thank Dr. Takashi Kumagai (Department of Neurosurgery, Yamagata Prefectural Central Hospital) and Dr. Kazuhiro Ando (Department of Neurosurgery, Brain Research Institute, Niigata University) for their initial help with this case report. We also thank Editage for assistance with English language editing. 


\section{References}

1. Bakay L, Leslie EV. Surgical treatment of vertebral artery insufficiency caused by cervical spondylosis. J Neurosurg. 1965; 23(6):596-602.

2. Nagashima C. Surgical treatment of vertebral artery insufficiency caused by cervical spondylosis. J Neurosurg. 1970;32(5):512-521.

3. Husni EA, Bell HS, Storer J. Mechanical occlusion of the vertebral artery. A new clinical concept. JAMA. 1966;196(6):475-478.

4. Jost GF, Dalley AT. Bow hunter's syndrome revisited: 2 new cases and literature review of 124 cases. Neurosurg Focus. 2015;38(4):E7.

5. Sorensen BF. Bow hunter's stroke. Neurosurgery. 1978;2(3): 259-261.

6. Choi KD, Choi JH, Kim JS, et al. Rotational vertebral artery occlusion: mechanisms and long-term outcome. Stroke. 2013; 44(7):1817-1824.

7. Grossmann RI, Davis KR. Positional occlusion of the vertebral artery: a rare cause of embolic stroke. Neuroradiology. 1982;23(4):227-230.

8. Andereggen L, Arnold M, Andres RH, et al. Bow hunter's stroke due to prominent degenerative spinal disorder. Clin Neuroradiol. 2012;22(4):355-358.

9. Okawa M, Amamoto T, Abe H, et al. Wake-up stroke in a young woman with rotational vertebral artery occlusion due to far-lateral cervical disc herniation. J Neurosurg Spine. 2015;23(2): 166-169.

10. Nishikawa H, Miya F, Kitano $\mathrm{Y}$, et al. Positional occlusion of vertebral artery due to cervical spondylosis as rare cause of wakeup stroke: report of two cases. World Neurosurg. 2017;98: 877.e13-877.e21.

11. Matsuyama T, Morimoto T, Sakaki T. Bow hunter's stroke caused by a nondominant vertebral artery occlusion: case report. Neurosurgery. 1997;41(6):1393-1395.

12. Yeh JF, Lin YJ, Po HL, et al. A case of bow hunter's stroke caused by non-dominant vertebral artery. Acta Neurol Taiwan. 2005;14(2): 69-73.

13. Noh Y, Kwon OK, Kim HJ, et al. Rotational vertebral artery syndrome due to compression of nondominant vertebral artery terminating in posterior inferior cerebellar artery. J Neurol. 2011; 258(10):1775-1780.

14. lida $\mathrm{Y}$, Murata $\mathrm{H}$, Johkura $\mathrm{K}$, et al. Bow hunter's syndrome by nondominant vertebral artery compression: a case report, literature review, and significance of downbeat nystagmus as the diagnostic clue. World Neurosurg. 2018;111:367-372.

15. Choi JH, Kim MJ, Lee TH, et al. Dominant vertebral artery occlusion during ipsilateral head tilt. Neurology. 2011;76(19):1679.

16. Kuether TA, Nesbit GM, Clark WM, et al. Rotational vertebral artery occlusion: a mechanism of vertebrobasilar insufficiency. Neurosurgery. 1997;41(2):427-433.
17. Wang $Y$, Wang $Y$, Zhao $X$, et al. Clopidogrel with aspirin in acute minor stroke or transient ischemic attack. N Engl J Med. 2013; 369(1):11-19.

18. Topçuoglu MA, Arsava EM, Ay H. Antiplatelet resistance in stroke. Expert Rev Neurother. 2011;11(2):251-263.

19. Lu DC, Zador Z, Mummaneni PV, et al. Rotational vertebral artery occlusion-series of 9 cases. Neurosurgery. 2010;67(4): 1066-1072.

20. Vilela MD, Goodkin R, Lundin DA, et al. Rotational vertebrobasilar ischemia: hemodynamic assessment and surgical treatment. Neurosurgery. 2005;56(1):36-45.

21. Zaidi HA, Albuquerque FC, Chowdhry SA, et al. Diagnosis and management of bow hunter's syndrome: 15-year experience at Barrow Neurological Institute. World Neurosurg. 2014;82(5): 733-738.

22. Kan $P$, Yashar $P$, Langer DJ, et al. Posterior inferior cerebellar artery to posterior inferior cerebellar artery in situ bypass for the treatment of bow hunter's-type dynamic ischemia in holovertebral dissection. World Neurosurg. 2012;78(5): 553.e15-553.e17.

23. Sugiu K, Agari T, Tokunaga K, et al. Endovascular treatment for bow hunter's syndrome: case report. Minim Invasive Neurosurg. 2009;52(4):193-195.

24. Mori K, Ishikawa K, Fukui I, et al. A subtype of bow hunter's syndrome requiring specific method for detection: a case of recurrent posterior circulation embolism due to "hidden bow hunter's syndrome." JNET J Neuronendovasc Ther. 2018;12(6): 295-302.

\section{Disclosures}

The authors report no conflict of interest concerning the materials or methods used in this study or the findings specified in this paper.

\section{Author Contributions}

Conception and design: Nozawa, Okamoto, Nakazato, Morita, Fujii. Acquisition of data: Motohashi, Tashi, Fujii. Analysis and interpretation of data: Okamoto, K Watanabe, Fujii. Drafting the article: Okamoto, Hasegawa, Fujii. Critically revising the article: Okamoto, Fujii. Reviewed submitted version of manuscript: Okamoto, Suzuki, Tashi, Hasegawa, M Watanabe, Kawashima, Fujii. Administrative/ technical/material support: K Watanabe, Kawashima. Study supervision: Okamoto, Hasegawa, Kawashima, Fujii.

\section{Correspondence}

Takanori Nozawa: Yamagata Prefectural Central Hospital, Yamagata City, Japan. tnozawa@bri.niigata-u.ac.jp. 